\title{
Stable gravastars—an alternative to black holes?
}

\author{
Matt Visser $^{1}$ and David L Wiltshire ${ }^{2}$ \\ ${ }^{1}$ School of Mathematical and Computing Sciences, Victoria University of Wellington, \\ New Zealand \\ 2 Department of Physics and Astronomy, University of Canterbury, Private Bag 4800, \\ Christchurch, New Zealand \\ E-mail: matt.visser@vuw.ac.nz and d.wiltshire@phys.canterbury.ac.nz \\ Received \\ Published DD MMM 2003 \\ Online at stacks.iop.org/CQG/21/1 (DOI: 10.1088/0264-9381/21/0/000)
}

\begin{abstract}
The 'gravastar' picture developed by Mazur and Mottola is one of a very small number of serious challenges to our usual conception of a 'black hole'. In the gravastar picture there is effectively a phase transition at/ near where the event horizon would have been expected to form, and the interior of what would have been the black hole is replaced by a segment of de Sitter space. While Mazur and Mottola were able to argue for the thermodynamic stability of their configuration, the question of dynamic stability against spherically symmetric perturbations of the matter or gravity fields remains somewhat obscure. In this paper we construct a model that shares the key features of the Mazur-Mottola scenario, and which is sufficiently simple for a full dynamical analysis. We find that there are some physically reasonable equations of state for the transition layer that lead to stability.
\end{abstract}

PACS number:

\section{Introduction}

Whereas researchers in the relativity community (and the bulk of the astrophysics and particle physics communities) are by and large happy with our understanding of classical black holes, there is a certain amount of polite dissent. Such dissent ranges from a careful sceptical analysis of what it physically means to observe an event horizon [1], through alternative models for compact objects [2], to more radical proposals that drastically modify the physics in the region where the event horizon would otherwise be expected to form [3-8].

In particular, the 'gravastar' (gravitational vacuum star) picture recently developed by Mazur and Mottola [8] is one of a very small number of serious challenges to our usual conception of a 'black hole'. In the gravastar picture there is effectively a phase transition at or near the location where the event horizon would have been expected to form. The interior of what would have been the black hole is replaced by a suitably chosen segment of 
de Sitter space. (See also Laughlin et al [5-7] for a similar proposal.) While Mazur and Mottola were able to make considerable progress with their proposal, and to argue for the thermodynamic stability of their configuration, the question of whether their model enjoys full dynamic stability against spherically symmetric perturbations of the matter and/ or gravity fields remains an open question. In this paper we construct a simplified model that shares the key features of the Mazur-Mottola scenario, and which is sufficiently simple to be amenable to a full dynamical analysis. We find that there are some physical equations of state for the transition layer that lead to stability.

\section{The physical model}

To set the stage, we note that the Mottola-Mazur gravastar is an onion-like construction which has five layers (including two thin shells):

- An external Schwarzschild vacuum, with energy density, $\rho=0$, and pressure, $P=0$.

- A thin shell, with surface density $\sigma_{+}$and surface tension $\vartheta_{+}$; with radius $r_{+} \gtrsim 2 M$.

- A (relatively thin) finite-thickness shell of stiff matter with equation of state $P=\rho$; straddling $r=2 M$ where the horizon would in normal circumstances have formed.

- A second thin shell; with radius $r_{-} \lesssim 2 M$, and with surface density $\sigma_{-}$and surface tension $\vartheta_{-}$.

- A de Sitter interior, with $P=-\rho$.

The two thin shells are used to 'confine' the stiff matter in a transition layer straddling $r=2 M$, while the energy density in the de Sitter vacuum is chosen to satisfy

$$
\frac{4 \pi}{3} \rho(2 M)^{3}=M
$$

so that, in the approximation where the transition layer is neglected, all of the mass of the resulting object can be traced back to the energy density of the de Sitter vacuum.

In order to dynamically study the gravitational stability of this setup, we will simplify the model even further. We shall combine the thick shell of stiff matter, and the two thin shells, into a single thin shell. This reduces the gravastar to a simple three-layer model:

- An external Schwarzschild vacuum, $\rho=0=p$.

- A single thin shell, with surface density $\sigma$ and surface tension $\vartheta$; with radius $a \gtrsim 2 M$.

- A de Sitter interior, $P=-\rho$.

To avoid forming an event horizon, we shall demand

$$
\frac{4 \pi}{3} \rho(2 M)^{3} \lesssim M
$$

The closer we are to saturating this bound, the closer we will be to the Mazur-Mottola scenario (and the closer we will be to attributing all the mass of the gravastar to the energy of the de Sitter vacuum).

We will analyse the dynamical stability of this simplified three-layer model. Initially, we had hoped to either unambiguously determine stability or instability (which would then have killed the gravastar scenario). The actual results are much more ambiguous: some physically reasonable equations of state for the thin shell lead to stability, but that situation does not appear to be completely generic_-some fine tuning seems to be necessary. 


\section{The mathematical model}

Let us consider the class of geometries

$$
\mathrm{d} s^{2}=-\left[1-\frac{2 m(r)}{r}\right] \mathrm{d} t^{2}+\frac{\mathrm{d} r^{2}}{1-2 m(r) / r}+r^{2}\left(\mathrm{~d} \theta^{2}+\sin ^{2} \theta \mathrm{d} \phi^{2}\right) .
$$

While less general than the class of all static spherically symmetric geometries, this restricted class of metrics is more than sufficient for our current needs, and includes both the Schwarzschild and the de Sitter geometries. We will assume that two geometries of this type are connected along a timelike hypersurface at $r=a(t)$, with spacelike normal, $n^{a}$. By considering a point at fixed $\theta$ and $\phi$ and making use of the definition

$$
-\mathrm{d} \tau^{2}=-\left[1-\frac{2 m(a(t))}{a(t)}\right] \mathrm{d} t^{2}+\frac{1}{1-2 m(a(t)) / a(t)}\left[\frac{\mathrm{d} a(t)}{\mathrm{d} t}\right]^{2} \mathrm{~d} t^{2},
$$

we can reparametrize the position of the timelike hypersurface in terms of $\tau$ - the proper time along this hypersurface - and so determine $a(\tau)$. To understand the dynamics of the hypersurface we adopt the Israel-Lanczos-Sen thin-shell formalism [9]. The induced metric on the shell,

$$
h_{a b}=g_{a b}-n_{a} n_{b},
$$

is given by

$$
h_{a b} \mathrm{~d} x^{a} \mathrm{~d} x^{b}=-\mathrm{d} \tau^{2}+a(\tau)^{2}\left(\mathrm{~d} \theta^{2}+\sin ^{2} \theta \mathrm{d} \phi^{2}\right) .
$$

We are interested in the extrinsic curvature

$$
K_{a b}=h_{a}{ }^{c} h_{b}{ }^{d} \nabla_{c} n_{d}
$$

so that we can then apply the junction conditions to relate the discontinuity in extrinsic curvature to the surface stress-energy, $S_{a b}$, located on the shell:

$$
\left[\left[K_{a b}\right]\right]=-8 \pi\left[S_{a b}-\frac{1}{2} S h_{a b}\right], \quad\left[\left[K_{a b}-K h_{a b}\right]\right]=-8 \pi S_{a b} .
$$

Here $[[X]]$ denotes the discontinuity in $X$ across the shell. A modern exposition of the thinshell formalism (in a rather different physical context) can be found in [10] to which we make extensive reference in the interests of simplifying the current presentation.

\subsection{Static shell}

For simplicity, let us first assume the shell is static. The extrinsic curvature may be computed directly from definition (7), or alternatively in terms of Gaussian normal coordinates (GNC). Define $n^{a}=(\partial / \partial \eta)^{a}$, where

$$
\mathrm{d} \eta=\frac{\mathrm{d} r}{\sqrt{1-2 m(r) / r}} .
$$

In GNC the extrinsic curvature is

$$
K_{a b}=\frac{1}{2} \frac{\partial g_{a b}}{\partial r} \frac{\partial r}{\partial \eta},
$$

or in an orthonormal basis,

$$
K_{\hat{a} \hat{b}}=\sqrt{1-2 m(a) / a} \operatorname{diag}\left(\frac{\{m(a) / a\}^{\prime}}{1-2 m(a) / a}, \frac{1}{a}, \frac{1}{a}\right) .
$$


Then in an orthonormal frame the surface stress-energy tensor is given by $S_{\hat{a} \hat{b}}=$ $\operatorname{diag}(\sigma,-\vartheta,-\vartheta)$, and standard manipulations yield both

$$
\left[\left[\sqrt{1-2 m(a) / a} a^{-1}\right]\right]=-4 \pi \sigma,
$$

and

$$
\left[\left[\frac{1-m(a) / a-m^{\prime}(a)}{a \sqrt{1-2 m(a) / a}}\right]\right]=-8 \pi \vartheta .
$$

Thus there are only two algebraically independent components of the extrinsic curvature and they can be related to surface density and surface tension. (Compare, for instance, with [10], pp $179 \mathrm{ff}^{3}$ For calculationally similar though physically distinct presentations, see also [11-15].)

Equation (12) may be recast as

$$
\sqrt{1-2 m_{+} / a}=\sqrt{1-2 m_{-} / a}-4 \pi \sigma a,
$$

where we use the notation $m_{+} \equiv m\left(a_{+}\right)$and $m_{-} \equiv m\left(a_{-}\right)$. If we square both sides of equation (14) and rearrange it follows that

$$
8 \pi \sigma a^{2} \sqrt{1-2 m_{-} / a}=16 \pi^{2} \sigma^{2} a^{3}+2\left(m_{+}-m_{-}\right) .
$$

A further manipulation of this sort then yields

$$
16 \pi^{2} \sigma^{2} a^{4}+4 m_{+} m_{-}=\left[8 \pi^{2} \sigma^{2} a^{3}+\left(m_{+}+m_{-}\right)\right]^{2} .
$$

If we wish to solve (16) for $a$ as a function of the other parameters, we are dealing with a sextic. In contrast, solving for any one of $m_{+}, m_{-}$, or $\sigma$ is no worse than a simple quadratic. One can simplify equation (16) by expressing it in terms of $m_{s}=4 \pi \sigma a^{2}$, which represents the mass of the thin shell itself. We then obtain a (static) 'master equation', which directly relates the three masses $\left(m_{+}, m_{-}\right.$and $\left.m_{s}\right)$ to the radial location of the shell, $a$ :

$$
m_{s}^{2}+4 m_{+} m_{-}=\left[m_{s}^{2} /(2 a)+\left(m_{+}+m_{-}\right)\right]^{2} .
$$

We have gone through this analysis in some detail as we will want to re-use and generalize this calculation in the dynamic case.

The master equation (17) is only one single equation; one would simultaneously have to solve the surface tension equation. In many ways the best approach for the static shell is prescriptive: choose the interior and exterior geometries arbitrarily, then choose $a$, and then calculate the shell energy density $\sigma$ and surface tension $\vartheta$ from equations (12) and (13). Of course, this analysis presently gives no information about stability; we are simply assuming a static shell. Developing a dynamical stability analysis is our next concern.

\subsection{Dynamic shell}

We now add time dependence by allowing the shell to move radially, so that the proper time, $\tau$, at points on the shell is given by (4). It follows from (4) that the (unit) 4-velocity of a point on the hypersurface (at fixed $\theta$ and $\phi$ ) is

$$
V^{a}=\left(\frac{\sqrt{\Delta+\dot{a}^{2}}}{\Delta}, \dot{a}, 0,0\right),
$$

3 Note that equation (15.39b) on page 179 of [10] has a typographical error and should read

$$
\vartheta=-\frac{1}{8 \pi}\left\{-\kappa^{\hat{\imath} \hat{\imath}}+\kappa^{\hat{\theta} \hat{\theta}}\right\} \text {. }
$$


where $\Delta \equiv 1-2 m(a) / a, \dot{a}=\mathrm{d} a / \mathrm{d} \tau$ and we have chosen $\tau$ to be future-pointing with respect to $t$. The unit normal to the shell is obtained from (18) using the orthogonality condition, $n_{a} V^{a}=0$, giving

$$
n^{a}=\left(\frac{\dot{a}}{\Delta}, \sqrt{\Delta+\dot{a}^{2}}, 0,0\right) .
$$

There is an overall sign ambiguity in defining the components of the unit normal (18), which is fixed by demanding that the chart of the Gaussian normal coordinates $(\tau, \eta)$ be consistently oriented with respect to the chart of local coordinates $(t, r)$, the Jacobian of the transformation being

$$
\frac{\partial(\tau, \eta)}{\partial(t, r)}=\left(\begin{array}{cc}
\sqrt{\Delta+\dot{a}^{2}} & -\Delta^{-1} \dot{a} \\
-\dot{a} & \Delta^{-1} \sqrt{\Delta+\dot{a}^{2}}
\end{array}\right),
$$

with unit determinant and inverse

$$
\frac{\partial(t, r)}{\partial(\tau, \eta)}=\left(\begin{array}{cc}
\Delta^{-1} \sqrt{\Delta+\dot{a}^{2}} & \Delta^{-1} \dot{a} \\
\dot{a} & \sqrt{\Delta+\dot{a}^{2}}
\end{array}\right) .
$$

The components of the extrinsic curvature in GNC may be computed using (10) and (21), or alternatively directly from definition (7). In fact, the second approach is more direct in the present case. On account of the orthogonality relation, combined with the definition of the induced metric, we have $h_{a}{ }^{b} V^{a}=V^{b}$, so that it immediately follows that

$$
\begin{aligned}
K_{\hat{\imath} \hat{\imath}}=K_{\tau \tau} & =V^{a} V^{b} K_{a b} \\
& =V^{a} V^{b} \nabla_{a} n_{b} \\
& =-n_{b} A^{b},
\end{aligned}
$$

where $A^{b} \equiv V^{a} \nabla_{a} V^{b}$ is the 4-acceleration of a point on the shell. Since the 4-acceleration and 4-velocity are orthogonal we must have $A^{b}=A n^{b}$, so that

$$
K_{\hat{\imath} \hat{\imath}}=-A,
$$

where $A$ is the magnitude of the 4-acceleration of the shell. The angular components of the extrinsic curvature are easily found to be

$$
K_{\hat{\theta} \hat{\theta}}=K_{\hat{\phi} \hat{\phi}}=\frac{1}{a} \sqrt{\Delta+\dot{a}^{2}} .
$$

Imposing the junction conditions, similarly to the case of the static shell, we then have

$$
\sigma=-\frac{1}{4 \pi a}\left[\left[\sqrt{1-2 m(a) / a+\dot{a}^{2}}\right]\right]
$$

and

$$
\vartheta=-\frac{1}{8 \pi}\left[\left[\frac{\sqrt{1-2 m(a) / a+\dot{a}^{2}}}{a}+A\right]\right] .
$$

Since the restricted class of geometries we are considering possesses a timelike Killing vector, $k^{a}=(\partial / \partial t)^{a}$, the 4-acceleration is easily calculated. Following [10, p 183], we can use both

$$
\frac{\mathrm{d}}{\mathrm{d} \tau}\left(k_{a} V^{a}\right)=-A \dot{a}
$$

and

$$
\frac{\mathrm{d}}{\mathrm{d} \tau}\left(k_{a} V^{a}\right)=-\frac{\mathrm{d}}{\mathrm{d} \tau}\left\{\sqrt{1-2 m / a+\dot{a}^{2}}\right\}
$$


to give the simple relation

$$
A=\frac{\ddot{a}-\{m / a\}^{\prime}}{\sqrt{1-2 m / a+\dot{a}^{2}}} .
$$

This gives the 4-acceleration of the shell in terms of a combination of kinematic $(\ddot{a}, \dot{a})$ and gravitational $(m(a) / a)$ properties. Substituting (29) in (26) we have

$$
\vartheta=-\frac{1}{8 \pi a}\left[\left[\frac{1-m / a-m^{\prime}+\dot{a}^{2}+a \ddot{a}}{\sqrt{1-2 m / a+\dot{a}^{2}}}\right]\right] .
$$

We now have explicit formulae for both surface density (25) and surface tension (30). These equations are the natural generalization of equations (15.63) and (15.64) of [10], where a somewhat more restricted class of metrics was considered in a different context.

\subsection{Conservation laws}

Equation (30) for $\vartheta$, though manageable, is still a little messy. There is no need to use it directly, however, since equations (25) and (30) are related on account of energy-momentum conservation. It is easily verified by straightforward manipulations of (25) and (30) that

$$
\frac{\mathrm{d}}{\mathrm{d} \tau}\left(\sigma a^{2}\right)=\vartheta \frac{\mathrm{d}}{\mathrm{d} \tau}\left(a^{2}\right) .
$$

We note that in general one would expect the rhs of (31) to be supplemented by a term of the form

$$
\text { flux term }=\left[\left[T_{a b} V^{a} n^{b}\right]\right],
$$

corresponding to the net discontinuity in the momentum flux, $F_{a}=T_{a b} V^{b}$, impinging on the shell. Such a term is absent in the case of the special class of metrics (3) considered here, for which

$$
\rho=-p_{r}=\frac{1}{4 \pi} \frac{m^{\prime}}{r^{2}},
$$

and

$$
p_{t}=-\frac{1}{8 \pi} \frac{m^{\prime \prime}}{r} .
$$

Since the density equals minus the radial pressure, when restricted to the $r-t$ plane $T_{a b} \propto g_{a b}$. But since both $V$ and $n$ lie in the $r-t$ plane this implies

$$
T_{a b} V^{a} n^{b} \propto g_{a b} V^{a} n^{b}=0,
$$

and the flux terms on both sides of the shell automatically vanish. This is a special feature of the 'sufficiently general' class of geometries (3). By contrast, in the general spherically symmetric geometry this flux term would be somewhat difficult to deal with.

Consequently, if we are provided with an equation of state for the shell $\sigma=\sigma(\vartheta)$, (or $\vartheta=\vartheta(\sigma)$ ), we can formally integrate the conservation equation (31) to determine $\sigma(a)$ as a function of $a$. Once we have that function, all the dynamics will be encoded in the single dynamical equation (25), the result being true subject only to our 'general enough' restriction on metric (3). 


\section{The master equation}

\subsection{Derivation}

Let us re-write the dynamical $\sigma$ equation (25) as

$$
\left[\left[\sqrt{1-2 m(a) / a+\dot{a}^{2}}\right]\right]=-4 \pi \sigma(a) a,
$$

where we are now keeping arbitrary time dependence in the form of $\dot{a}$. That is

$$
\sqrt{1-2 m_{+}(a) / a+\dot{a}^{2}}=\sqrt{1-2 m_{-}(a) / a+\dot{a}^{2}}-4 \pi \sigma(a) a .
$$

A series of steps identical to those that lead from (14) to (17) now yields

$$
m_{s}^{2}\left(1+\dot{a}^{2}\right)+4 m_{+} m_{-}=\left[\frac{m_{s}^{2}}{2 a}+\left(m_{+}+m_{-}\right)\right]^{2},
$$

where as before $m_{s}=4 \pi \sigma a^{2}$ is the mass of the thin shell. This dynamic 'master equation' is of the form of an 'energy equation' for a nonrelativistic particle,

$$
\frac{1}{2} \dot{a}^{2}+V(a)=E,
$$

with 'potential'

$$
V(a)=\frac{1}{2}\left\{1+\frac{4 m_{+}(a) m_{-}(a)}{m_{s}^{2}(a)}-\left[\frac{m_{s}(a)}{2 a}+\frac{\left(m_{+}(a)+m_{-}(a)\right)}{m_{s}(a)}\right]^{2}\right\},
$$

and 'energy' $E=0$.

There will then be a strictly stable solution for the shell (stable against spherically symmetric radial oscillations) if and only if there is some $m_{s}(a)$ and some $a_{0}$ such that we simultaneously have

$$
V\left(a_{0}\right)=0, \quad V^{\prime}\left(a_{0}\right)=0, \quad V^{\prime \prime}\left(a_{0}\right)>0 .
$$

A small quirk in this relativistic calculation is that since $E \equiv 0$, the situation where $V(a) \equiv 0$, which in nonrelativistic mechanics corresponds to neutral equilibrium, is now converted to a situation of stable equilibrium (since now, because one is not free to increase the 'energy' $E$, one has $\dot{a} \equiv 0$ ).

There is a less stringent notion of stability that is also useful, that of 'bounded excursion'. Suppose we have $a_{2}>a_{1}$ such that

$$
V\left(a_{1}\right)=0, \quad V^{\prime}\left(a_{1}\right) \leqslant 0, \quad V\left(a_{2}\right)=0, \quad V^{\prime}\left(a_{2}\right) \geqslant 0
$$

with $V(a)<0$ for $a \in\left(a_{1}, a_{2}\right)$. In this situation the motion of the shell remains bounded by the interval $\left(a_{1}, a_{2}\right)$. Although not strictly stable, since the shell does in fact move, this notion of 'bounded excursion' more accurately reflects some of the aspects of stability naturally arising in nonrelativistic mechanics. In particular, it is simply a version of the standard stability criterion for orbits about the fixed point that would exist if we were free to arbitrarily specify the constant $E$ on the rhs of (39), and corresponds to orbits about the fixed point which are 'stable but not asymptotically stable'. In the present context, perturbing the potential by adding a small negative offset

$$
V(a) \rightarrow V(a)-\epsilon^{2}
$$

will generically convert a strictly stable potential to one exhibiting 'bounded excursion'. 


\subsection{Inverting the potential}

Suppose we now take the potential $V(a)$, and the masses $m_{ \pm}(a)$ as given. Then equation (40) yields a quadratic equation for $m_{s}^{2}(a)$ :

$$
\begin{aligned}
m_{s}^{2}(a)=2 a^{2}\{ & 1-2 V(a)-\frac{m_{+}(a)}{a}-\frac{m_{-}(a)}{a} \\
& \left. \pm \sqrt{1-2 V(a)-\frac{2 m_{+}(a)}{a}} \sqrt{1-2 V(a)-\frac{2 m_{-}(a)}{a}}\right\} .
\end{aligned}
$$

It is important to note that we have not chosen any specific equation of state for the shell; we have only used the assumption that some equation of state exists. As always when solving a quadratic, there is a risk that one of the roots is unphysical. In this case the (+) sign is unphysical and the $(-)$ sign the physically interesting branch. This may most easily be verified by setting $m_{+}(a)=m_{-}(a)$ in which case the spacetime geometry is completely continuous across the location of the shell, so that its mass $m_{s}$ (and surface energy density $\sigma$ and surface tension $\vartheta$ ) must be zero. Therefore the surface energy density $\sigma(a)$ is given by the expression

$$
\begin{aligned}
& \sigma(a)= \\
& \pm \frac{\sqrt{2}}{4 \pi a} \sqrt{1-2 V(a)-\frac{m_{+}(a)}{a}-\frac{m_{-}(a)}{a}-\sqrt{1-2 V(a)-\frac{2 m_{+}(a)}{a}} \sqrt{1-2 V(a)-\frac{2 m_{-}(a)}{a}}} .
\end{aligned}
$$

We can now, by noting that the contents of the outer radical sign above are a perfect square, extract the square root. The overall sign is determined by the unique choice which leads to the above expression being the compatibility equation between equations (39) and (36). This now yields

$$
\begin{aligned}
\sigma(a) & =-\frac{1}{4 \pi a}\left\{\sqrt{1-2 V(a)-\frac{2 m_{+}(a)}{a}}-\sqrt{1-2 V(a)-\frac{2 m_{-}(a)}{a}}\right\} \\
& =-\frac{1}{4 \pi a}\left[\left[\sqrt{1-2 V(a)-\frac{2 m(a)}{a}}\right]\right] .
\end{aligned}
$$

There is a subtlety one should be aware of when comparing equation (36) with (47). Whereas in (36) we are simply determining the energy density on a shell if it happens to have 'velocity' $\dot{a}$ at radius $a$, here in (47) we are determining something considerably more powerful based on a fully dynamic analysis, namely, what the surface energy density would have to be as a function of $a$ in order to be compatible with the specified potential $V(a)$. Proceeding further, from the conservation equation

$$
\vartheta=\sigma+\frac{1}{2} a \frac{\mathrm{d} \sigma}{\mathrm{d} a}
$$

we can calculate $\vartheta(a)$. Analytically, the result is

$$
\vartheta(a)=-\frac{1}{8 \pi a}\left[\left[\frac{1-2 V(a)-m(a) / a-a V^{\prime}(a)-m^{\prime}(a)}{\sqrt{1-2 V(a)-2 m(a) / a}}\right]\right] .
$$

Recall that $m_{ \pm}(a)$ and $V(a)$ are at this stage arbitrary functions that we are still free to prescribe. These expressions for $\sigma(a)$ and $\vartheta(a)$ - equations (47) and (49)-now determine 
the equation of state parametrically. In summary

- Either we choose an equation of state in the form of a specific $\sigma(\vartheta)$ and integrate the conservation equation to determine $\sigma(a)$ (and hence $m_{s}(a)$ and hence $V(a)$ );

- $o r$ we choose a potential in the form $V(a)$ and parametrically extract the corresponding equation of state using the algorithm just presented.

\subsection{Specialization of gravastar geometry}

We will now specialize to a specific model for the gravastar, which simplifies the MazurMottola model to a thin shell separating two bulk regions:

- Outside, a Schwarzschild region $T_{a b}=0$.

- Inside, a de Sitter region $T_{a b} \propto g_{a b}$.

Thus $m_{+}$and $m_{-}$are no longer arbitrary. In view of our assumptions we can simply write $m_{+}=M$, the total external mass of the system, while

$$
m_{-}=(4 \pi / 3) \rho a^{3}=k a^{3},
$$

where $\rho$ is the energy density in the de Sitter vacuum and we have introduced the parameter $k$ simply to minimize the explicit appearance of factors of $4 \pi / 3$. Keeping $V(a)$ arbitrary, our previous analysis implies

$$
\sigma(a) \equiv \frac{1}{4 \pi a}\left\{\sqrt{1-2 V(a)-2 k a^{2}}-\sqrt{1-2 V(a)-\frac{2 M}{a}}\right\},
$$

while for $\vartheta(a)$ we have

$\vartheta(a) \equiv \frac{1}{8 \pi a}\left\{\frac{1-2 V(a)-a V^{\prime}(a)-4 k a^{2}}{\sqrt{1-2 V(a)-2 k a^{2}}}-\frac{1-2 V(a)-a V^{\prime}(a)-M / a}{\sqrt{1-2 V(a)-2 M / a}}\right\}$.

These are now a pair of parametric equations determining the equation of state for any three-layer Mazur-Mottola model with exterior Schwarzschild geometry and interior de Sitter geometry in terms of a freely specified $V(a)$. (For formally similar analyses in physically different contexts, see $[14,15]$.) If we want the system to be strictly stable at some $a_{0}$ then we need to enforce equation (41). There are two obvious examples of such behaviour:

- $V(a) \equiv 0$, a degenerate, but physically important, case corresponding to $\dot{a} \equiv 0$.

- $V(a)=\frac{1}{2}\left(a-a_{0}\right)^{2} f(a)$, where $f(a)$ is an arbitrary positive function which is regular at $a_{0}$. In this situation the stability is in a sense trivial: the master equation has a unique solution at $a=a_{0}$ and $\dot{a}=0$, and all possibility of motion is excluded by fiat.

More generally we could consider cases of 'bounded excursion' by choosing $V(a)=$ $\frac{1}{2}\left(a-a_{0}\right)^{2} f(a)-\epsilon^{2}$. For sufficiently small $\epsilon$ there will be distinct roots $a_{1}$ and $a_{2}$ where $V(a)=0$, with $a_{1}<a_{0}<a_{2}$. The motion of the shell will be of 'bounded excursion' and forced to remain in the interval $\left(a_{1}, a_{2}\right)$.

These observations are now enough to guarantee that there are large classes of potential $V(a)$ - and so large classes of equation of state $\sigma(\vartheta)$-for which the system is stable against radial oscillations. Of course there are also large classes of potential for which the system is unstable. Depending on one's views regarding the gravastar model this may be interpreted as either good news or bad news. 


\subsection{The $V(a) \equiv 0$ case}

We will now investigate the $V(a) \equiv 0$ case. This is an important case as it corresponds to a completely static gravastar with $\dot{a} \equiv 0$. Furthermore, since equations (51) and (52) have no explicit dependence on $V^{\prime \prime}(a)$ or higher derivatives of $V$, the analysis we will present suffices to determine the relationship between the energy density, $\sigma$, and surface tension, $\vartheta$, of the shell at any specific $a_{0}$ for which $V\left(a_{0}\right)=0$ and $V^{\prime}\left(a_{0}\right)=0$.

Setting $V(a) \equiv 0$ in (51) and (52) we find

$$
\sigma(a) \equiv \frac{1}{4 \pi a}\left\{\sqrt{1-2 k a^{2}}-\sqrt{1-\frac{2 M}{a}}\right\},
$$

and

$$
\vartheta(a) \equiv \frac{1}{8 \pi a}\left\{\frac{1-4 k a^{2}}{\sqrt{1-2 k a^{2}}}-\frac{1-M / a}{\sqrt{1-2 M / a}}\right\} .
$$

It is easy to check that the strong energy condition

$$
\sigma-2 \vartheta>0
$$

is satisfied for all values of the parameters for which real solutions exist. The null energy condition is consequently also satisfied. However, other energy conditions are possibly violated. (We note that there are good reasons for not taking the energy conditions too seriously as fundamental physics [16]. Nevertheless, the energy conditions are still useful as a crude measure of how peculiar a certain matter distribution is.)

Since we assume $M>0$ in order that $\sigma$ and $\vartheta$ be real, we require $a>2 M$ and $a \leqslant 1 / \sqrt{2 k}$. Consequently $k<1 /\left(8 M^{2}\right)$. The surface energy function $\sigma(a)$ ranges from the positive value

$$
\sigma(2 M)=\frac{\sqrt{1-8 k M^{2}}}{8 \pi M},
$$

at $a=2 M$, to the negative value

$$
\sigma\left(\frac{1}{\sqrt{2 k}}\right)=\frac{-1}{4 \pi} \sqrt{2 k(1-2 M \sqrt{2 k})},
$$

at $a=1 / \sqrt{2 k}$, and crosses zero at $a=(M / k)^{1 / 3}$. Thus the weak energy condition is satisfied for $2 M<a<(M / k)^{1 / 3}$, but violated for $(M / k)^{1 / 3} \leqslant a<1 / \sqrt{2 k}$.

The status of the dominant energy condition, $|\vartheta| \leqslant \sigma$, is more complicated and depends crucially on the value of the dimensionless parameter $k M^{2}$. The surface tension $\vartheta$ itself is negative on the whole interval $2 M<a<1 / \sqrt{2 k}$, and diverges as $\vartheta \rightarrow-\infty$ at the endpoints. To get the full equation of state $\vartheta(\sigma)$ we can simply obtain a graphical solution from (53) and (54) by plotting $\theta(a)$ versus $\sigma(a)$ parametrically.

As an example, we provide several plots exploring the case $k M^{2}=1 / 18$, for which $a_{\min }=2 M$ and $a_{\max }=1 / \sqrt{2 k}=3 M$. In this case the dominant energy condition is violated throughout the entire range $a \in(2 M, 3 M)$.

As a second specific example we plot the case $k M^{2}=1 / 72$, for which $a_{\max }=$ $1 / \sqrt{2 k}=6 M$, in figure 5. In this case the dominant energy condition is satisfied for $2.1243194234 M \leqslant a \leqslant 3 M$, and violated throughout the rest of the range $a \in(2 M, 6 M)$. The 'least stiff' equation of state for this example is obtained at $a=2.5764849609 M$ when $\vartheta=-0.6912774322 \sigma$. We have simply shown the central region of the equation of state $\sigma(\vartheta)$ in figure 5, since the functions $\sigma(a)$ and $\vartheta(a)$ have the same qualitative form as the case depicted in figures 1 and 2, the only difference being that $a$ now ranges from $a=2 M$ to $a=6 M$. 


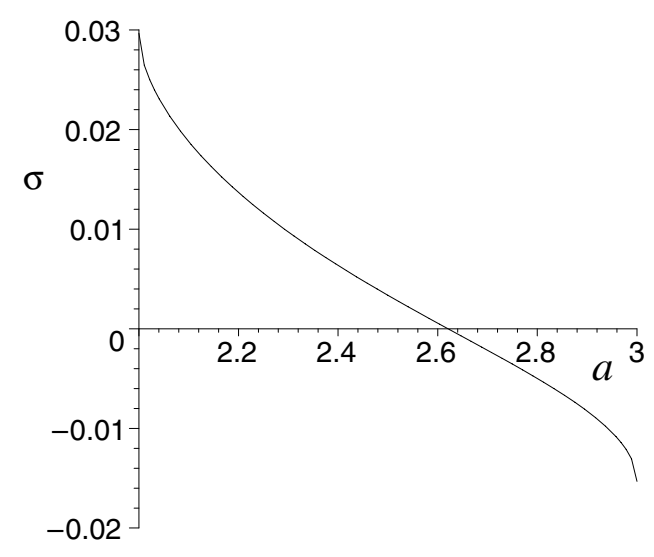

Figure 1. Surface energy density, $\sigma$ (in units $M^{-1}$ ), as a function of radius, $a$ (in units $M$ ). $\left(k M^{2}=1 / 18, V(a) \equiv 0.\right)$

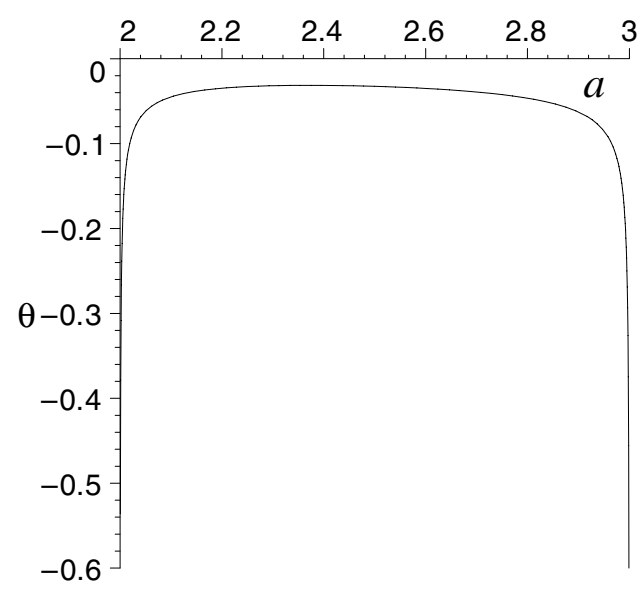

Figure 2. Surface tension, $\vartheta$ (in units $M^{-1}$ ), as a function of radius, $a$ (in units $\left.M\right) .\left(k M^{2}=1 / 18\right.$, $V(a) \equiv 0$.

The range of parameter values for which it is possible to obtain stable configurations which satisfy the dominant energy condition can be quantified precisely. The critical case is that of a stiff matter shell, for which $P=\rho$, or $\sigma=-\vartheta$. Applying this condition to (51) and (52) we have

$$
\left(3-\frac{5 M}{a}\right) \sqrt{1-2 k a^{2}}=\left(3-8 k a^{2}\right) \sqrt{1-\frac{2 M}{a}} .
$$

If we square both sides of (58) and rearrange we obtain a sextic in $a$,

$64 k^{2} a^{6}-128 k^{2} M a^{5}-30 k a^{4}+36 k M a^{3}+50 k M^{2} a^{2}+12 M a-25 M^{2}=0$.

Only half of the roots of (59) are potentially of interest since (59) also applies to the equation one would obtain by changing the relative sign of the roots on each side of (58). Given that the lhs of (58) is positive for $a>2 M$, positivity of the rhs of (58) further tightens the upper bound on $a$ to $a<\sqrt{3} / \sqrt{8 k}$. There are then at most two real solutions of (59) in the range $2 M<a<\sqrt{3} / \sqrt{8 k}$. (Note that in order for this range to be non-empty we must have 


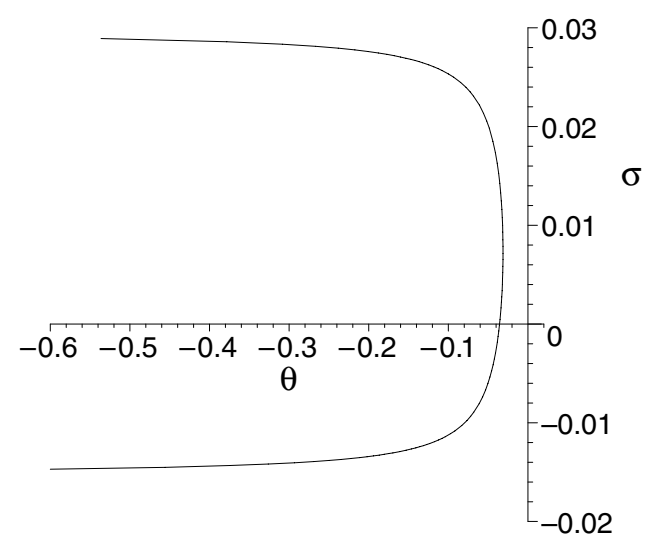

Figure 3. Equation of state: surface energy density as a function of surface tension. $\left(k M^{2}=1 / 18\right.$, $V(a) \equiv 0$.

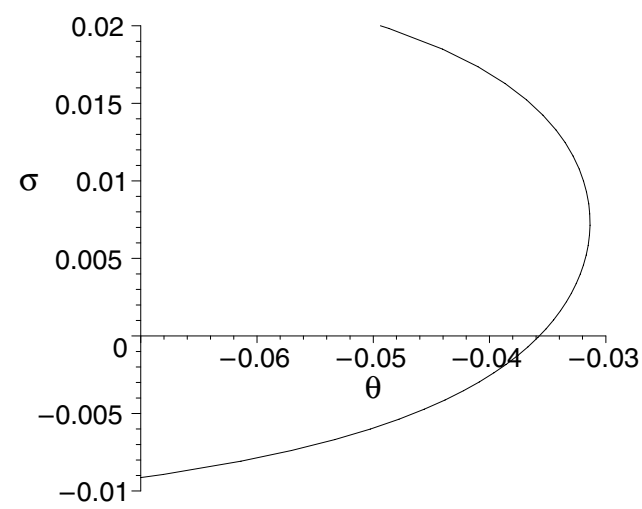

Figure 4. Equation of state: enlargement of the central region of figure 3-surface energy density as a function of surface tension. $\left(k M^{2}=1 / 18, V(a) \equiv 0\right.$.)

$k M^{2}<3 / 32$, which is why the dominant energy condition was always violated in our first example.)

To derive the condition for the existence of the relevant thin shells it is convenient to rewrite (59) in terms of the dimensionless parameters $\alpha=a / M$ and $\lambda=k M^{2}$, so that

$$
64 \lambda^{2} \alpha^{6}-128 \lambda^{2} \alpha^{5}-30 \lambda \alpha^{4}+36 \lambda \alpha^{3}+50 \lambda \alpha^{2}+12 \alpha-25=0 .
$$

Solutions of the required type exist if the discriminant of (60), when considered as a polynomial in $\alpha$, is negative, i.e.,

$$
-14155776 \lambda^{7}(200 \lambda-27)^{2} D_{4}(\lambda)<0,
$$

where

$D_{4}(\lambda) \equiv\left(400000000 \lambda^{4}-1054320000 \lambda^{3}+257041039 \lambda^{2}-19516500 \lambda+337500\right)$.

This means that the quartic $D_{4}(\lambda)$ must be positive. Solving for roots of this quartic numerically we find that

$$
\lambda=k M^{2} \leqslant \lambda_{\text {cr }}=0.0243045493773
$$




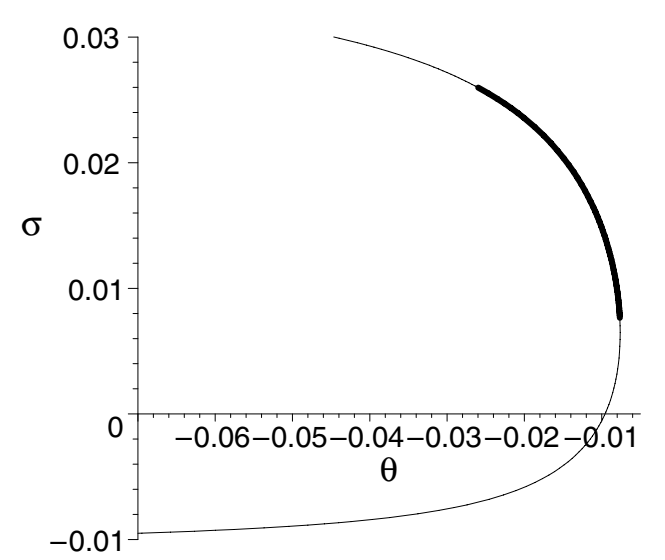

Figure 5. Equation of state: enlargement of the central region for an example in which the dominant energy condition is satisfied. Surface energy density as a function of surface tension for the case $k M^{2}=1 / 72 ; V(a) \equiv 0$. Parameter values for which the dominant energy condition is violated are shown by a thin line, and parameter values for which the dominant energy condition is satisfied, namely $2.124319 M<a<3 M$, are shown by a thick line.

is the critical condition for the existence of thin shells which satisfy the dominant energy condition.

In the critical case $k M^{2}=\lambda_{\text {cr }}$ the dominant energy condition is satisfied only for a stiff matter thin shell at $a=a_{\mathrm{cr}} \equiv 2.3005600972496 M$. For parameter values $0<k M^{2}<\lambda_{\mathrm{cr}}$, there will be a range of values $a_{1}<a<a_{2}$ over which the dominant energy condition is satisfied. Figure 5 shows one such example. The bounds $a_{1,2}$ are given by the relevant solutions of (59), such that $2 M<a_{1}<a_{\mathrm{cr}}$ and $a_{\mathrm{cr}}<a_{2}<\sqrt{3} / \sqrt{8 k}$. Thin shells with $a=a_{1,2}$ will have stiff matter equations of state, while those with $a_{1}<a<a_{2}$ will have 'almost stiff' equations of state. Since the dominant energy condition is the most stringent of the standard energy conditions this construction implies that all the standard energy conditions will be satisfied.

Although the construction used in this section has not yielded an analytic expression for the equation of state in a form $\sigma(\vartheta)$, it has nevertheless been 'èffective'- this construction does certainly demonstrate that there are physically reasonable equations of state that lead to a stable gravastar configuration for many values of the parameters $a, M$ and $k$.

It is also important to note that the $V(a) \equiv 0$ examples considered in this section are notable mainly for their relative simplicity-there is no physical reason for not choosing $V(a) \neq 0$, say $V(a)=\frac{1}{2}\left(a-a_{0}\right)^{2} f(a)-\epsilon^{2}$, and doing so opens new possibilities for stable shell configurations.

\section{Special gravastar geometries}

\section{1. $V \equiv 0$ equation of state—special limits}

For a general equation of state of the thin shell, two special limits of the $V \equiv 0$ model are of interest:

- $k \rightarrow 0$, corresponding to an empty interior.

- $k \rightarrow 1 /\left(8 M^{2}\right)$, corresponding to the Mazur-Mottola limit. 
For an empty interior, results are straightforward, and can be explicitly written as

$$
\begin{aligned}
& \sigma(a)=\frac{1-\sqrt{1-2 M / a}}{4 \pi a}, \\
& \vartheta(a)=\frac{1}{8 \pi a}\left[1-\frac{1-M / a}{\sqrt{1-2 M / a}}\right] .
\end{aligned}
$$

Though these formulae are very similar to standard results occurring in the theory of thin shells, the interpretation here is rather different-remember, these are the $\sigma(a)$ and $\vartheta(a)$ required to have $V(a) \equiv 0$; and so a transition layer that is dynamically stable (to radial perturbations) at all $a$.

In contrast the Mazur-Mottola model corresponds to another particular limiting choice for the energy density in the de Sitter vacuum:

$$
k(2 M)^{3}=M \quad \Rightarrow \quad k=\frac{1}{8 M^{2}} .
$$

To understand the nature of this limit it is convenient to write

$$
k=\frac{1}{8 M^{2}(1+\epsilon)^{2}}
$$

with $\epsilon \gtrsim 0$. Then the energy density and surface tension in (53) and (54) are both real for

$$
a \in(2 M, 2 M[1+\epsilon]) .
$$

This indicates, purely on kinematic grounds, a severely restricted range of possible motions for the shell.

In the limit that $\epsilon \rightarrow 0$ the surface energy density of our three-layer model is forced to go to zero. In the language of the five-layer Mazur-Mottola model [8] this means

$$
\sigma_{\text {net }}=\sigma_{+}+\rho_{\text {transition }} \eta_{\text {transition }}+\sigma_{-} \rightarrow 0 \text {. }
$$

In other words, as the transition layer in the Mazur-Mottola five-layer model becomes thinner and thinner, the contributions from $\sigma_{ \pm}$conspire with the density of the stiff matter in the transition layer to produce a net surface energy density of zero. A quick way of seeing that this must be the case is to realize that if one is attributing all of the mass of the black hole to the de Sitter vacuum in the interior, then whatever is going on in the transition layer must average out to zero surface energy density.

In contrast, the transverse pressures in the transition layer do not have any pleasantly behaved limit. Indeed $\vartheta \rightarrow-\infty$, corresponding to infinite transverse pressures. This is not unexpected, and is one of the reasons many in the general relativity community are extremely hesitant to adopt models with thin layers of matter hovering just at the location where the event horizon would have been expected to form.

\subsection{Stiff-shell gravastars}

Given the potential problems associated with the limits just discussed, there is another model among the class we have considered which might be considered to be an alternative three-layer simplification of the Mazur-Mottola model. In particular, let us specify the equation of state of the thin shell to be that of stiff matter, $P=\rho$ or $\vartheta=-\sigma$. This is a limit of the Mazur-Mottola model in which the equation of state of the thick stiff matter shell is maintained, but the shell itself is made infinitesimally thin. One principle difference is that no attempt is made to equate 
the energy of the interior de Sitter vacuum with the ADM mass of the exterior solution. The other crucial point is that the thin shell is to be placed at $a>2 M$, where one avoids infinite transverse pressures discussed above.

The analysis of the conditions required for satisfying the dominant energy condition in section 4.4 has already shown that such solutions exist and are dynamically stable to spherically symmetric perturbations. This follows since the stiff matter shell occurs at the extreme values for which the dominant energy condition holds. In particular, we found that such solutions exist if $k M^{2}$ is less than or equal to the critical value given by (63). Furthermore, for $\lambda<\lambda_{\text {cr }}$ there are two values of $a$ at which we can place a stiff shell in stable gravastars, the lower value, $a_{1}$, being in the range $2 M<a_{1}<2.30056 M$. For the case plotted in figure 5 , for example, $a_{1}=2.12432 M$ and $a_{2}=3 M$. The inner stiff shell case is certainly so close to the putative horizon that any test to distinguish such an object from a true Schwarzschild black hole would be extremely difficult in astrophysical contexts.

\subsection{Anti-de Sitter gravastars}

Although we have primarily been interested in the case of a de Sitter interior, our general analysis remains valid for the case of an anti-de Sitter interior, with $\Lambda<0$. For such interiors it is impossible to satisfy the junction conditions with a thin shell having a stiff equation of state. However, it is possible to take a thin shell with a 'cosmological constant' or 'vacuum energy' equation of state, $P=-\rho$, or $\vartheta=+\sigma$.

Since $k<0$, there are a number of changes to the analysis of section 4.4. In particular, there is now no upper bound to the value of $a$. The surface energy, $\sigma$, is always positive, being bounded below by (56). The strong energy condition is now violated for particular parameter values. We are interested in the particular case $\vartheta=\sigma$. Applying this condition to (51) and (52) we have

$$
\left(1-\frac{3 M}{a}\right) \sqrt{1-2 k a^{2}}=\sqrt{1-\frac{2 M}{a}} .
$$

Squaring (70) and rearranging we find that the shell must be located at values of $a$ which solve the quartic

$$
2 a^{4} k-12 a^{3} M k+18 M^{2} k a^{2}+4 M a-9 M^{2}=0
$$

and lie in the range $a>3 M$. An analysis of the discriminant of this quartic reveals that solutions exist for all values $k<0$. Furthermore, for each value of $k<0$ there is only one root of (70) in the range $a>3 M$. For small values of $-k M^{2}$ one finds that $a$ is very large, but as $-k M^{2}$ gets larger the value of $a$ approaches $3 M$ asymptotically. For $k M^{2}=-1$, for example, $a=3.44898 M$.

\section{Discussion}

The general relativity community is, by and large, extremely comfortable with the 'black hole' concept; a concept firmly based in the classical solutions of the Einstein equations [17-19]. In contrast, there is a strong undercurrent (certainly not the mainstream) in the particle physics and condensed matter communities that views the notion of event horizons with some alarm - this has led over the years to repeated suggestions that quantum physics should intervene at/ near the would-be event horizon, either replacing it with a singularity or 
preventing appearance of the horizon at the first place. A particular proposal along these lines that has recently attracted considerable attention is the Mazur-Mottola 'gravastar' [8].

In this paper we have considered the dynamical stability of a three-layer simplification of the five-layer Mazur-Mottola model. Aficionados of the model will be happy to know that there are many equations of state for the transition layer that imply dynamical stability for the gravastar configuration. Those who are less enamoured of the model will be equally happy to see that large classes of equation of state are ruled out. Our own interpretation of these results is that this calculation, or suitable modification thereof, is a necessary first step towards any kind of serious model building with a thin transition layer separating two 'vacuum' regions.

In this regard, we have in this paper explored a tentative suggestion: if one really wishes to build a three-layer thin-shell alternative to a black hole, then it seems to us that $r=2 \mathrm{M}$ is not the appropriate place to insert the transition layer. In order to avoid the infinite stress (presumably cutoff at the Planck scale so that we would really be talking about Planck-scale stresses) that occurs when the transition layer is placed at $r=2 M$, it would seem to us to be more profitable to move the transition layer out to $r>2 M$.

We have demonstrated that particular models do exist which realize this possibility. One particular case is the stiff shell gravastar, namely an exterior Schwarzschild geometry, and an interior de Sitter vacuum, separated by a stiff matter thin shell. Such models could be regarded as simplified versions of the Mazur-Mottola model which avoid its singular limit. Stiff shell gravastars exist and are dynamically stable for $\Lambda \leqslant 6 \lambda_{\text {cr }} / M^{2}=0.145827 / M^{2}$. In fact, generally two possible stiff shell gravastars exist, and in one of these cases one must place the thin shell at a value $2 M<r<2.30056 M$. Since this is very close to the nominal position of a black hole horizon, it would be extremely difficult to distinguish the exterior geometry of such a gravastar from a genuine black hole in astrophysical contexts.

The idea of moving the transition layer to a finite $r>2 M$ should possibly also be explored in the related Chapline-Hohlfeld-Laughlin-Santiago model [5-7]. More generally, if the transition layer is not 'thin' but rather of finite thickness, then investigations along the lines of Gliner's [20] and Dymnikova's [21] ideas might be profitable.

Finally we emphasize what we feel is the most important technical aspect of this paper. We have analysed spherically symmetric thin shells in a large class of background geometries (arbitrary $m(r)$ ), and related the resulting shell motion to an equivalent 'non-relativistic particle'. Furthermore, we have shown how to take any desired 'potential' $V(a)$ and 'invert' it to determine the equation of state for shell-matter that would lead to this potential. This result is of general interest to anyone building thin-shell models-regardless of one's views on gravastars versus black holes.

Note added: Since this paper was completed we have learned that solutions with features similar to those discussed here-possessing an asymptotically Schwarzschild exterior, a thin-shell and a de Sitter core-have recently been found by Wohlfarth [22] in a gravity model in which the Einstein-Hilbert action is replaced by a 'Born-Infeld style' action nonlinear in the curvature. Wohlfarth's solutions completely regularize the Schwarzschild singularity for $M>0$. While much work remains to be done, these results suggest that the model presented in this paper may represent a simplified version of a class of objects which occur very naturally in string theory inspired gravity models.

\section{Acknowledgments}

We would like to thank Benedict Carter for his careful reading and comments on an early version of the manuscript. This research was supported by the Marsden Fund administered by the Royal Society of New Zealand. 


\section{References}

[1] Abramowicz M A, Kluzniak W and Lasota J P 2002 No observational proof of the black-hole event-horizon Astron. Astrophys. 396 L31 (Preprint astro-ph/0207270)

[2] Mielke E W and Schunck F E 2000 Boson stars: alternatives to primordial black holes? Nucl. Phys. B 564185 (Preprint gr-qc/0001061)

[3] Stephens C R, 't Hooft G and Whiting B F 1994 Black hole evaporation without information loss Class. Quantum Grav. 11621 (Preprint gr-qc/9310006)

't Hooft G 1985 On the quantum structure of a black hole Nucl. Phys. B 256727

[4] Berezin V 2003 Black hole thermodynamics without a black hole? Nucl. Phys. B 661409 (Preprint gr-qc/ 0302066)

[5] Chapline G, Hohlfeld E, Laughlin R B and Santiago D I 2001 Quantum phase transitions and the breakdown of classical general relativity Phil. Mag. B 81235 (Preprint gr-qc/0012094)

[6] Chapline G, Laughlin R B and Santiago D I Emergent relativity and the physics of black hole horizons, Artificial Black Holes ed M Novello, M Visser and G Volovik (Singapore: World Scientific)

[7] Laughlin R B 2003 Emergent relativity Int. J. Mod. Phys. A 18831 (Preprint gr-qc/0302028)

[8] Mazur P O and Mottola E 2001 Gravitational condensate stars: an alternative to black holes Preprint gr-qc/ 0109035

[9] Israel W 1966 Singular hypersurfaces and thin shells in general relativity Nuovo Cimento B 44S10 1

Israel W 1967 Nuovo Cimento B 48463 (erratum)

Lanczos K 1924 Flächenhafte Verteilung der Materie in der Einsteinschen Gravitationstheorie Ann. Phys., Lpz 74518

Sen N 1924 Uber die Grenzbedingungen des Schwerefelds an unstetig Keistflächen Ann. Phys., Lpz 73365

[10] Visser M 1995 Lorentzian Wormholes: from Einstein To Hawking (New York: AIP now Springer-Verlag)

[11] Visser M 1989 Traversable wormholes from surgically modified Schwarzschild space-times Nucl. Phys. B 328 203

[12] Barcelo C and Visser M 2000 Brane surgery: energy conditions, traversable wormholes, and voids Nucl. Phys. B 584415 (Preprint hep-th/0004022)

[13] Barcelo C and Visser M 2000 Living on the edge: cosmology on the boundary of anti-de Sitter space Phys. Lett. B 482183 (Preprint hep-th/0004056)

[14] Brady P R, Louko J and Poisson E 1991 Stability of a shell around a black hole Phys. Rev. D 441891

[15] Poisson E and Visser M 1995 Thin shell wormholes: linearization stability Phys. Rev. D 527318 (Preprint gr-qc/9506083)

[16] Barcelo C and Visser M 2002 Twilight for the energy conditions Int. J. Mod. Phys. D 11 1553 (Preprint gr-qc/0205066)

[17] Thorne K S, Price R H and Macdonald D A 1986 Black Holes: The Membrane Paradigm (New Haven, CT: Yale University Press)

[18] Wald R M 1984 General Relativity (Chicago, IL: University of Chicago Press)

[19] Schutz B F 1895 A First Course In General Relativity (Cambridge: Cambridge University Press)

[20] Gliner E B 1998 Scalar black holes Preprint gr-qc/9808042

Gliner E B 2002 Inflationary universe and the vacuumlike state of physical medium Phys. Usp. 45213

Gliner E B 2002 Usp. Fiz. Nauk 45221

[21] Dymnikova I 2002 Cosmological term as a source of mass Class. Quantum Grav. 19725 (Preprint grqc/0112052)

Dymnikova I G 2000 The algebraic structure of a cosmological term in spherically symmetric solutions Phys. Lett. B 47233 (Preprint gr-qc/9912116)

Dymnikova I 1992 Vacuum nonsingular black hole Gen. Rel. Grav. 24235

[22] Wohlfarth M N R 2003 Gravity à la Born-Infeld Preprint hep-th/0310067 


\section{Endnotes}

(1) Author: Please provide PACS numbers in full form.

(2) Author: Please cite figures 3 and 4 in the text. 